\title{
Preventing Childhood, Infant and Neonatal Morbidities and Mortalities, What Interventions We Need
}

\author{
Hamid Yahya Hussain* \\ Professor of Community and Family Medicine, UAE \\ Received: January 04, 2018; Published: January 10, 2018 \\ *Corresponding author: Hamid Yahya Hussain, Professor of Community and Family Medicine, UAE
}

\section{Editorial}

The continuing of high rates of child mortality each and every year at national, regional and global levels from preventable causes: diarrhea, pneumonia, measles, malaria, HIV/AIDS, the underlying cause of under nutrition, is keep stigmating the global health efforts . Specifically, the one related child survival in lowincome settings and the interventions feasible for delivery at high coverage and high quality of required health care. It has been evidenced based that, greatest risk of childhood death occurs during the neonatal period, that extends from birth through the first month of life. As almost 60 percent of all deaths to children under age 5 and nearly two-thirds of infant deaths (birth to 12 months) occur during the neonatal period. Current available statistics revealed that about 98 percent of neonatal deaths take place in the developing countries. South Asia, stay as the highest annual neonatal mortality rates, where about 51 deaths occur for every 1,000 live births every year. About 2 million children were estimated die within a month of their birth. And 42 deaths occur in Africa, while in Latin America 25 in Latin America, and fewer than (10) in Europe and North America. As concerning neonatal morbidity in developing countries, the burden is unknown; yet, some recent studies of neonatal morbidity revealed high-risk (with a case fatality greater than 10 percent), about three-quarters experienced low-risk morbidities.

Current data base shows that, most neonatal deaths occur at home, following unsupervised deliveries; and limited epidemiological research indicates that the principal direct causes of neonatal death are infectious diseases, birth asphyxia, birth injuries, and the consequences of preterm birth and birth defects. At very early neonatal period (0-7 days), the major causes of death are asphyxia, infection, complications of prematurity, and birth defects; while infections cause majority of late neonatal deaths (8-28 days). In infants under 3 months of age in developing countries, infections are the major cause of mortality and morbidity. Almost more than 20 percent of children born in developing countries acquire an infection during the neonatal period, leading to an estimated 30 to 40 percent of total neonatal deaths.
Acute respiratory infections are on the top of causes of the death, bacterial sepsis and/or meningitis, neonatal tetanus, and diarrhea. Maternal infections, including sexually transmitted diseases (STDs) such as HIV (the topic of and syphilis, can be transmitted to the fetus or newborn in utero, through contact during labor and delivery, and in some cases, through breastfeeding. Antibioticresistant pathogens keep growing problem in developing countries. Neonatal sepsis and reports of nosocomial outbreaks from a variety of countries demonstrate that the problem of antibiotic resistance is of global concern .The widespread availability of antibiotics and their indiscriminate and inappropriate use contributes to this problem, along with poor infection-control practices in hospitals. Most ARI deaths are due to pneumonia, which annually kills more than 3 million children under the age of 5 in developing countries, In preterm neonates of low birth weight, respiratory distress syndrome, due to surfactant deficiency, is a major risk for early death, tuberculosis still as one of leading infectious disease, kills more than 2 million people worldwide each year, including 250,000 child, diarrhea is responsible for approximately 3 percent of all neonatal deaths, about 4 to 7 million neonates born each year worldwide that require some form of resuscitation immediately after birth, approximately 1 million die and another million suffer serious consequences, birth injuries are responsible for 11 percent of neonatal deaths worldwide.

When it comes to required interventions, international medical literature proves that global coverage for most interventions is still below 50\%. Nerveless if adequate interventions on quantity and quality wise universally available, $63 \%$ of child deaths or even more could be easily prevented. Such findings prove that, such necessary lifesaving interventions are strongly needed to achieve the development goal of reducing child mortality by two-thirds by 2015 are available, but that they are not being delivered to the mothers and children who need them. Strategies are needed to reduce the risk of infection, as well as to encourage the judicious prescription and appropriate use of antibiotics in the community. Surveillance competency capacity and the transfer of surveillance information 
must be developed to determine both the global and local impact of resistant microorganisms and to identify interventions that can address this threat.

Strategies we need to prevent infant and neonatal morbidities and mortalities strategies along with the means to make them effective in each setting. Improving education and behavioral change among women, families, and health care providers would have a major impact on birth outcomes. For direct impact, emphasis must be given to the delivery of care: getting appropriate services to those who need them and doing so in a timely manner. Interventions to reduce neonatal mortality and morbidity are discussed below, with emphasis on antenatal care, care during labor and delivery, and care during the early days and weeks of life. Care During Labor, Delivery, and the Very Early Neonatal Period, Prevention and treatment of neonatal infections, Prevention and treatment of neonatal infections, Breastfeeding and neo natal immunization

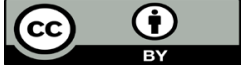

This work is licensed under Creative Commons Attribution 4.0 License

DOI: 10.32474/PAPN.2018.01.000103

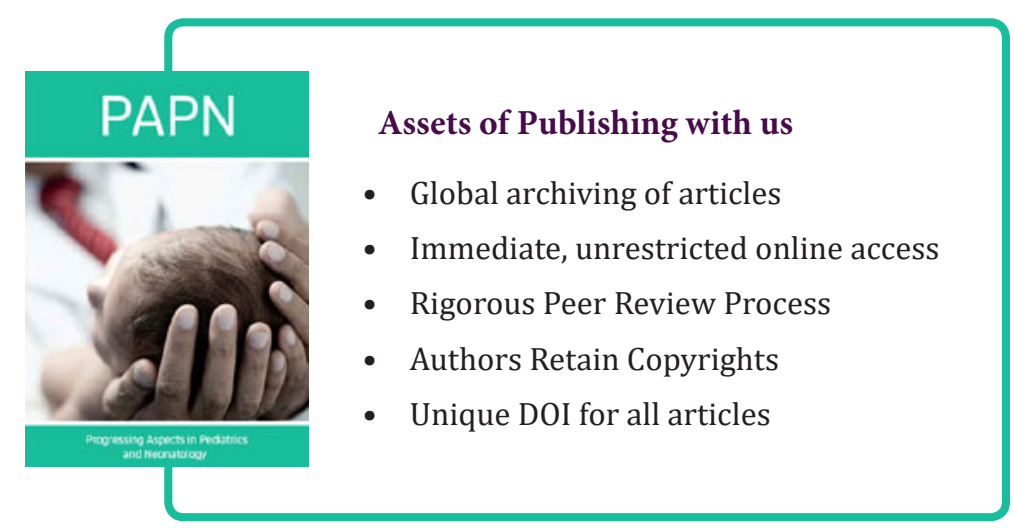

